\title{
Livewire Detector - Life Saver from Electrocution
}

\author{
K. Ramesh, B. Archana, V. Tharnath Reddy, C. Nagendra Kumar, M. Varaprasad
}

\begin{abstract}
Electrocution causes serious effect on human and animals and leads to dead some times. The Electrocution is one in all the main faced problems in forest areas and a few agricultural fields. Especially Forest guards were getting affected because of Electrocution in rainy seasons and through invisible condition. Forest guards get electrocuted in Forest due to electrical power lines and some of them lose their life by touching the electrical lines directly or when there is a leakage in supply lines. To find the electrocution condition, a device has been developed and is detailed in this article. This proposed device will detect the electrical power lines and give the alert signal to the user. The prototype model working has been verified at different work fields where the power lines present.
\end{abstract}

Key Words:Arduino Nano, Boost converter, Counter IC, signal receiver, battery level indicator, voltage display.

\section{INTRODUCTION}

India being an agricultural country, around 65\% people dependent on agriculture directly or indirectly. Because of stray animals, agricultural production is decreased by $30 \%$. To avoid it, farmers are using electrical fencing around their work field. If anyone is touching these electrical powered fencing, there may be a chance of losing their life and this kind of incident happened a lot in the past. In the past years, detection and prevention of downed conductors have been analyzed by several power industries and experts [1-7]. Since the live wires are not of producing arc every time and not giving any indications, it may cause serious danger to people or animals who touching it. Moreover, Frontline officers who are going to patrol in forest are suffering/losing their life when they touching the electrical power lines falling on the ground. The frontline of the forest department who guard our natural heritage also risk their lives, within the past forest guard shave lost their lives after they stepped on to live wires laid to kill animals in periphery of forests and national parks. This small device can help to save such lives. A small device which may be attached to a walking stick/ patrolling stick or be carried in pocket/backpack which will pontificate an alarm when it detects a sharply (domestic power supply line) within the vicinity (5m range).

Revised Manuscript Received on April 18, 2020

* Correspondence Author

Dr. K. Ramesh*, Professor and Head, Department of Electrical and Electronics Engineering, Kuppam Engineering College, India

B. Archana, Assistant Professor, Department of Electrical and Electronics Engineering, Kuppam Engineering College, India

V. Tharnath Reddy, UG Students, Department of Electrical and Electronics Engineering, Kuppam Engineering College, India

C.Nagendra Kumar, UG Students, Department of Electrical and Electronics Engineering, Kuppam Engineering College, India

M.Varaprasad, UG Students, Department of Electrical and Electronics Engineering, Kuppam Engineering College, India

(C) The Authors. Published by Blue Eyes Intelligence Engineering and Sciences Publication (BEIESP). This is an open access article under the CC BY-NC-ND license (http://creativecommons.org/licenses/by-nc-nd/4.0/)
During rainy season or in certain conditions, some electrical leakage may be happening in roadside electrical poles. People who are crossing this pole are affected when they are touching the pole. By using this proposed device, people will get alert before certain distance during their walk when they are crossing this kind of electrical poles. The proposed device has lot of potential usage and demand in the market. Based on the field of application, the device can be developed in different formats such as wearable model, pen type, and handheld device. The device has social responsibility in terms of saving human life from electrocution and is the motivation of this proposed work. Some of the potential areas are detailed below.

The proposed device can detect the presence of Power line placed inside a wall. Whenever, people doing alteration in houses, office, restaurants and etc., this device will help them to avoid the power lines, while making drill/ alterations in the wall. Some of the devices existing in the market are used to identify the AC live wires behind the walls, ceilings and floors. These devices having limitation in terms of detecting the live wire condition before certain distance. Other live wire detection devices designed using transistor models is producing the malfunction against the stones, water, wet soil or even by touching the sensor portion of the live wire detector. To avoid these conflicts, a new device has been proposed in this article and this will give the detection only with respect to live wire condition. In this article, a new live wire detector device has been detailed and compared its operation with transistor based model.

\section{LIVE WIRE DETECTION}

For the system of voltage detection there are sort of available existing solutions in the market. These are voltage detecting devices that are found in the forest trees to attain any absorption of voltage to the source. The indeed there are such difficulties need to be resolved in the existing method .For a device available in the market in terms of voltage detection not affordable to the forest guards (or) other use sin terms of protection. As a fared device it cannot be caused anywhere on the other hand, it is not a waterproof which is a needed feature with no lifetime duration for a protective device in an existing method in a discussion live wire coverage these is no availability of more than 3 meters which is most hazards to any users. Some of the devices available in the market are available with multi functionalities such as detection of metal rebar, stud and AC live wire and one of the device available in market is shown in Fig.1. The existing commercial devices are work well and gives almost accurate prediction about AC live wire condition behind the wall.

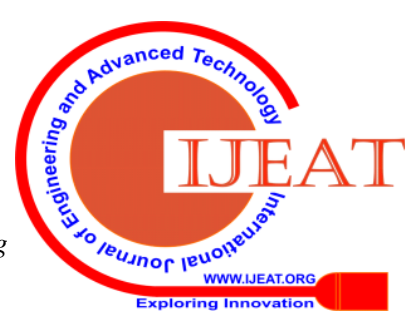



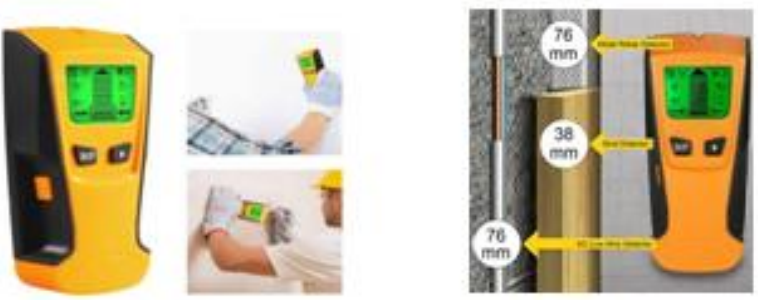

Fig.1 Existing Live Wire Detector

Forest guards get electrocuted in Forest due to electrical power lines and some of them lose their life by touching the electrical lines directly or when there is a leakage in supply lines. The proposed idea detailed in this article had won the first prize in the hackathon conducted by World Wide Fund (WWF) for Nation in association with Atal Incubation Centre - Sri Krishna devaraya University (SKU) on 18-06-2019. Later, the basic model of the proposed work has been developed as per the requirements given by the officials of World Wide Fund (WWF) for Nation, Jabalpur on December 07-08, 2019 to help the Forest guards to get the alert whenever the live wire is nearby.

This proposed device will detect the electrical power lines and give the alert signal to the user. The proposed idea is to

- Develop as a low cost handheld/ wearable device.

- Detect the power lines before minimum distance of 15-17feet

- Give alert signal through buzzer and/or vibration unit to the user

- Use an Emergency lighting system to see the Power line in the path during night time

- Use an additional electronic circuit for battery power indicator

The objectives of the proposed device are

- To detect the live wire condition and give the indication to the user before certain distance of a walk

- To avoid the loss of a human life due to electrocution

- To detect the harmful radiation from high power lines (As per studies which may lead to blood cancer)

The proposed idea consists of a small device that can be attached to a walking stick/ patrolling stick or be carried in pocket/back packet or used as a wearable device that can generate an alarm sound when it detects a live wire (domestic power supply line) in the vicinity (maximum of $5 \mathrm{~m}$ range).

\section{PROPOSED SYSTEM}

It consists of electronic circuit with an aerial and sense the A.C. Power line through electromagnetic induction process. Counter IC used in this circuit will generate the output whenever the AC power line is nearby. The proposed electronic circuit will generate the sound through buzzer arrangement whenever a live wire condition is detected. Emergency lighting system is proposed as an additional option in wearable model structure. The additional electronic circuit has been proposed to detect the charging level of the battery (used to power the live wire detector). The block diagram of the proposed Live Wire Detector device is detailed in Fig.2.

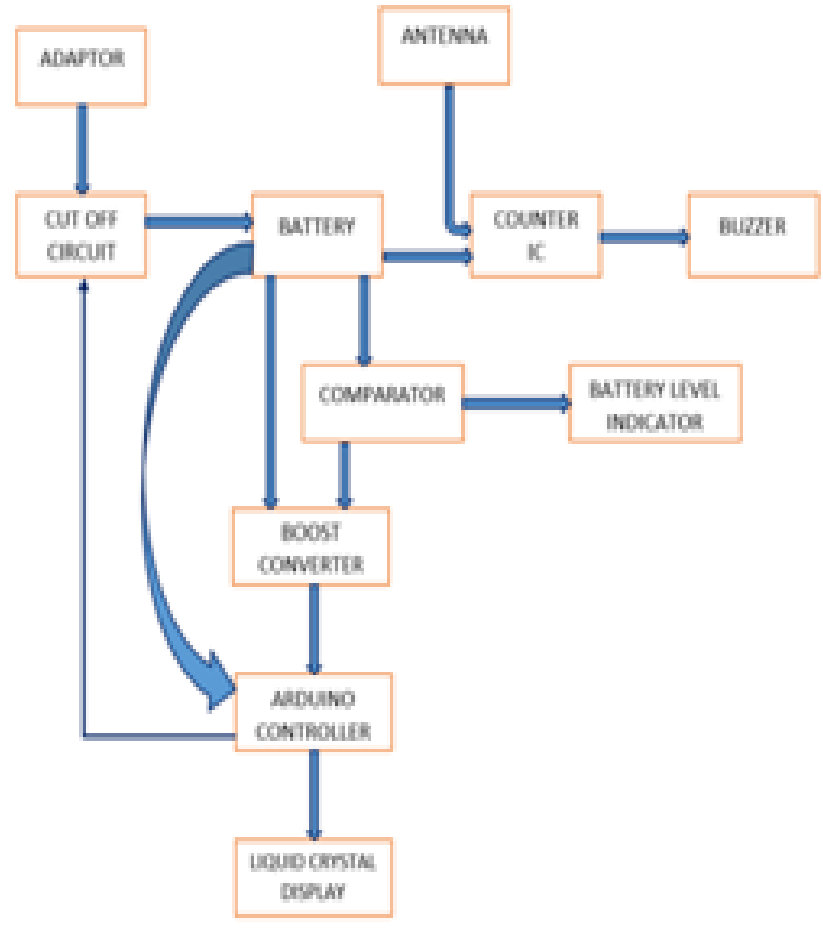

Fig.2Proposed Live Wire Detector

The proposed work is to detect voltage in live wire with the help of live wire detector to save the front-line officers lives. The proposed system consists of battery level indicator and antenna that sensing the voltage in certain distance of 5-6 meters and it is working with help of counter IC and when the antenna is sensing the voltage in live wire is will give sound with the help of buzzer. Arduino Nano used in this system is programmed with high powered embedded program to detect the voltage for a given range. Battery level indicator is mainly implanted in order to indicate the battery levels and percentage of battery is remained in the circuit. Battery level indicator is given with LED Strips which ensures each level of battery power. Boost converter is utilized in use to proper balance of voltage supply given to the circuit. LCD is important fact in the model which tends to perform and displays all activities of livewire detector .Buzzer is vital role in circuit which alerts the user with high voltage decibel for detection of high voltage. Counter IC model like IC547 is like heart of the circuit which monitors the livewire detector. These components are detailed in following sections.

\section{HARDWARE DESCRIPTION}

The proposed Live Wire Detector device consists of following major components namely

$>$ Live wire detection using counter IC

$>$ Antenna

> Battery level indication unit using LM3914

$>$ Emergency lighting unit

$>$ Battery over charging cutoff circuit

The proposed device has been developed as per the requirements given by WWF, Jabalpur and is developed as a prototype model and is detailed below.

Published By:

Blue Eyes Intelligence Engineering

\& Sciences Publication

(C) Copyriaht: All rights reserved.

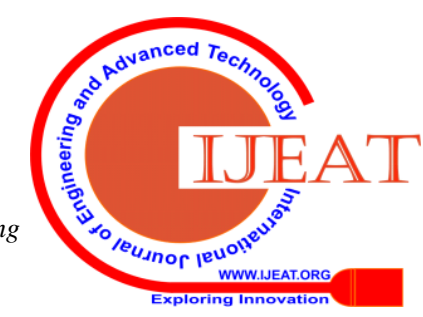


The circuit diagram of the proposed model is shown in Fig.3.

\subsection{Live wire detection using counter IC}

The proposed circuit diagram shown in Fig. 3. The proposed device using the counter IC (CD 4017) for the live wire detection process. It using an antenna and the antenna output is connected as an input to the counter IC at its Pin number 14 (through J3). Availability of the supply can be verified by pressing the switch $\mathrm{S} 1$ so that it will be indicated by glowing condition of LED L1. The input $(+3.7 \mathrm{~V})$ is applied using the battery B1 at the pin 16 of CD 4017. Whenever the antenna is nearby the power line, the counter IC will generate a clock signal at its output terminal based on the principal of electromagnetic induction. The output signal is amplified with the help of a transistor BC 547. The amplified output (available at collector terminal) is given to a buzzer and a LED. The buzzer (BUZ) sound and LED (L3) glowing condition indicates the detection of live wire. A resistor (R4) of $1 \mathrm{~K} \Omega$ is connected in series with L3 to limit the current flow. In this process, pin 13 and pin 15 of CD 4017 are connected to negative terminal of 3.7V, $3200 \mathrm{mAh}$ battery. Battery is selected with 3200mAh capacity to ensure long standing operating.

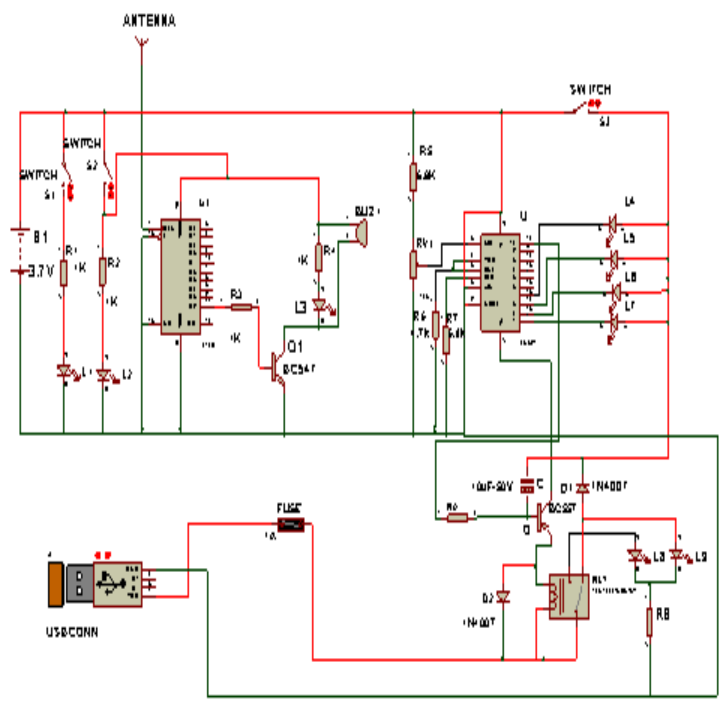

Fig.3 Circuit diagram of Live Wire Detector

\subsection{Antenna}

The antenna is designed by considering the copper wire of 22SWG by making more number of turns. The tail end of an antenna is connected to the CD 4017 at its pin 14. As an alternate option, the commercial antenna of Serial WIFI wireless Gain Antenna (shown in Fig. 4) can be used. With this antenna, the live wire condition can be detected before 5 meters.

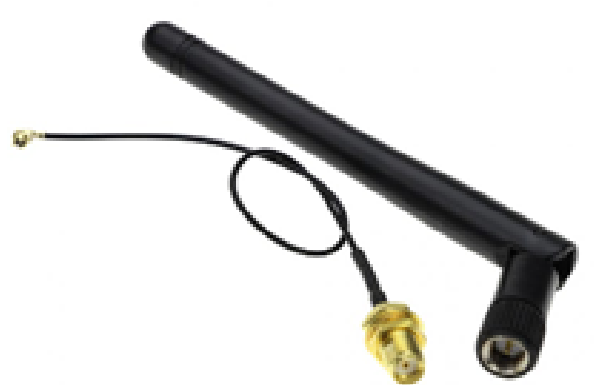

Fig.4 Serial WIFI wireless Gain Antenna

\subsection{Battery level indication unit using LM3914}

The LM 3914 is preferred for the battery level indication because of following options and it helps user to understand the battery voltage level.

$>$ Internal voltage reference from 1.2 to $12 \mathrm{v} \mathrm{DC}$.

$>$ Programmable output current $2 \mathrm{~mA}$ to $30 \mathrm{~mA}$.

$>$ LED driver outputs are current regulated.

$>$ No multiplexing interaction between outputs.

$>$ It supports wide range of temperature from 0 to 70 degree Celsius.

The proposed voltage level indicator unit uses R5 (6.8K $\Omega)$, RV1 (10K $\Omega)$, R6 $(4.7 \mathrm{~K} \Omega)$ and R7 (6.8K $\Omega)$ resistors to verify the battery voltage at different levels such as $25 \%, 50 \%, 75 \%$ and $100 \%$. A push switch S3 has to be pressed when the user wants to know the voltage level.

\subsection{Emergency lighting unit}

This unit consists of a LED lighting unit (with reflector) to provide the lighting during emergency condition (during night time). This unit is powered via a switch S1. In this operation, a current limiting resistor $(1 \mathrm{~K} \Omega)$ is connected in series with the LED (L1) to indicate battery ON condition.

\subsection{Battery over charging cut of circuit}

When the device is connected to power supply through an adopter device, the battery (will be connected via a relay (in normally closed operation). During this time, the D1 is in operation and the battery charging condition is indicated by L9 (LED). Once the battery is fully charged (around 4.2V), the relay will be deactivated with the help of resistor RA $(330 \Omega)$ and a capacitor of $10 \mu \mathrm{F}-50 \mathrm{~V}$ value. Once the relay is deactivated, it moves to normally open condition and L8 starts to glow (indicates the fully charged condition of battery). This circuit is used to avoid the overcharging of battery and ensures the battery safety. The adaptor circuit can be connected to the charging circuit across using USB port with a fuse of $1 \mathrm{~A}$ capacity to limit the current flow. The prototype model of Live Wire Detector has been developed and shown in Fig. 5.

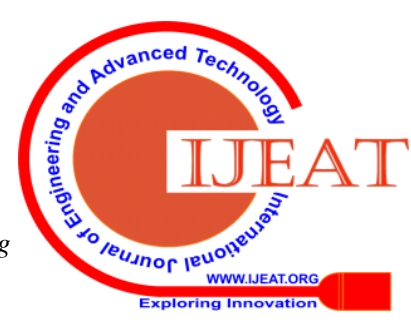




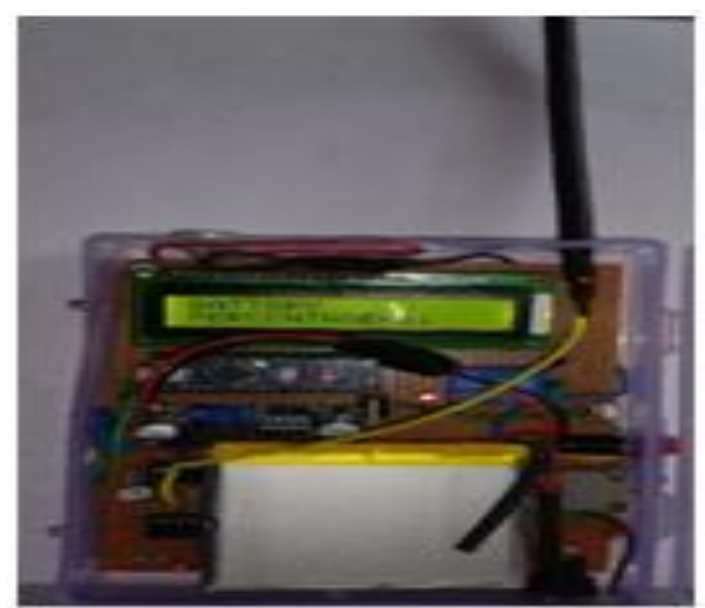

Fig. 5 Prototype model of Live Wire Detector

\section{RESULTS AND DISCUSSION}

The proposed prototype model operation has been verified with electrical power lines and domestic supply lines (Power cables, Junction boxes and etc.). The live wire testing process is shown in Fig. 6 and the distance at which the live wire condition is detected is noticed and detailed in Table 1.The same prototype model has been verified inside the rooms where the $220 \mathrm{~V}$ AC power lines are available. This proposed device will detect the AC power lines inside the wall almost 0.5 feet before and detect the AC power through the insulated cables before 1.5 feet. As compared to the existing devices in the market, the detection coverage distance is significantly more.

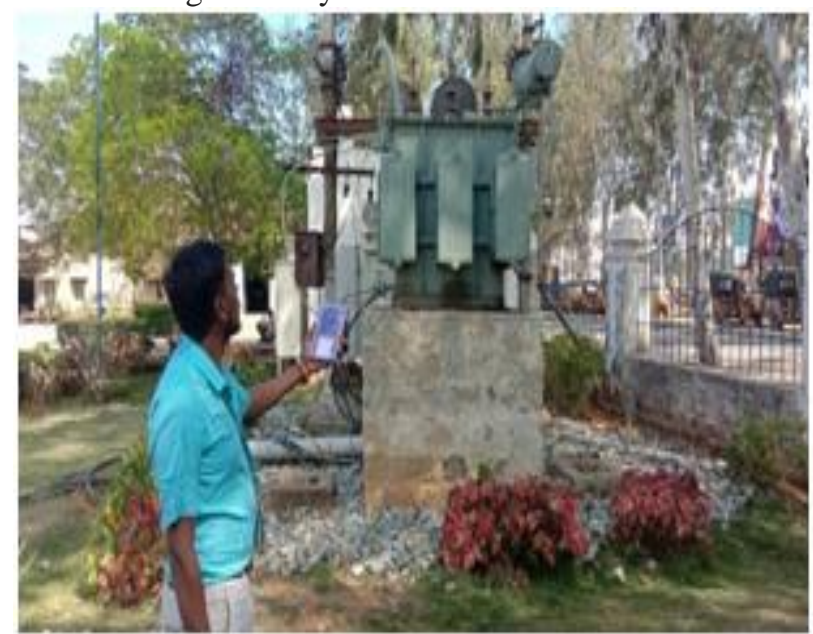

Fig. 6 Testing of Live Wire condition with proposed model (Venue: Kuppam Engineering College Campus)

Table 1 Distance at which detection of live wire condition details

\begin{tabular}{ccc}
\hline $\begin{array}{c}\text { Electrical point } \\
\text { (position) to be } \\
\text { sensed }\end{array}$ & $\begin{array}{c}\text { Distance covered } \\
\text { in Metre }\end{array}$ & $\begin{array}{c}\text { Distance covered in } \\
\text { Ft. }\end{array}$ \\
\hline $\begin{array}{c}220 \mathrm{~V} \text { Power } \\
\text { point/line }\end{array}$ & $1.524^{*}$ & 5 \\
\hline Transformer(440V) & 5 & 16.6 \\
\hline
\end{tabular}

The proposed device has been tested in the forest area of Jabalpur, Madhya Pradesh in front of WWF, Jabalpur officials and Bandhavgarh National park guards. Dr. K. Ramesh, Head of the Department, Electrical \& Electronics Engineering of Kuppam Engineering College demonstrated the prototype model with electrical fencing arrangement set by the Bandhavgarh National park in charges and the same are shown in Fig.7.

Uve Wire Detector demonstration at Jabalpur Forest office on Dec. 08, 2019
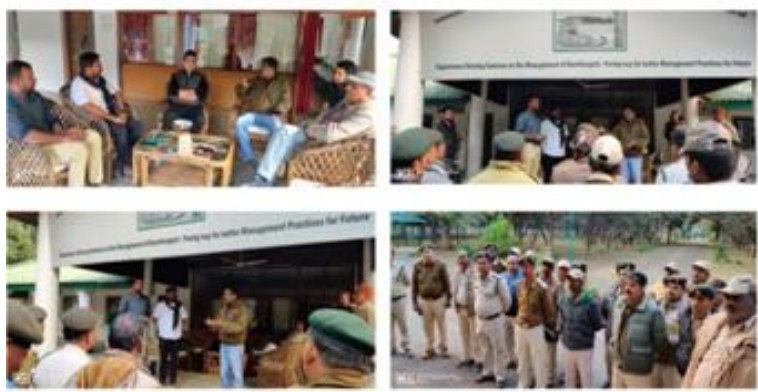

Fig.7 Prototype demonstration at Bandhavgarh Nation park, Jabalpur

After the successful demonstration of proposed Live Wire Detector device, few prototype models are sent for the field test at forest areas by WWF, Jabalpur authorities. The proposed work has been selected for the incubation work by World Wide Fund (WWF) for Nation in association with Atal Incubation Centre - SKU.

The proposed device has lot of potential usage and demand in the market. Based on the field of application, the device can be developed in different formats such as wearable model, pen type, and handheld device. The device has social responsibility in terms of saving human life from electrocution and is the motivation of this proposed work.

\section{CONCLUSION}

The proposed system is of low-cost device and the device is to carry easily in the hand and pocket. It is very useful for frontline officers and it save the lives of frontline staff of Forest Department who patrol the forest areas, National Parks \& Sanctuaries, Tiger \& Elephant Reserves. The proposed device accurately sensing the live wire condition and provides the $360^{\circ}$ coverage and is rugged in construction. The proposed device may help all common people to detect the electrical leakage/electrocution condition especially in rainy condition. Malfunctions happening in Transistor based live wire detection process are completely removed in this proposed device.

\section{ACKNOWLEGEMENT}

Authors thank the Management of Kuppam Engineering College, World Wide Fund (WWF) for Nation, Jabalpurand Atal Incubation Centre - SKU for their support in developing the proposed device.

\section{REFERENCES}

1. Dr.S.P. Vimal , M. Tamilarasan, T.Balaji, C.B.Aravind babu, "Wireless AC Line Detector", International Journal of Scientific \& Engineering Research Volume 8, Issue 7, July-2017 ISSN 2229-5518

\section{Published By:}

Blue Eyes Intelligence Engineering \& Sciences Publication

(C) Copyriaht: All rights reserved.

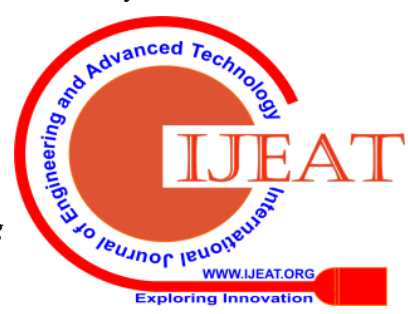


2. P.Priya, R.Karuppasamy, R.RagavaRaja, B. Pradeep, "Design of Wireless Networked Electricity Pole Line Multi-Fault MonitoringSystem", International Journal of Recent Technology and Engineering (IJRTE) ISSN: 2277-3878, Vol. 8, No. 4S2, 2019.

3. V. Sonandkar, A. Bhati, D. Gupta, S. Chouhan, N. Kinhekar and N. P. Padhy, "Power measurement using arduino for effective demand response", IEEE 6th International Conference on Power Systems (ICPS), New Delhi, 2016, pp. 1-5.

4. Sachil Kumar, Anoop K. Verma, Uma Shankar Singh, "Electrocutionrelated mortality in northern India - A5-year retrospective study", Egyptian Journal of Forensic Sciences, Vol. 4, pp. 1-6, 2014.

5. Yasar Tirasci, Suleyman Goren, Mehmet Subasi, Fuat Gurkan, "Electrocution-Related Mortality: A Review of 123 Deaths in Diyarbakir, Turkey between 1996 and 2002", Volume 208 Issue 2 Pages 141-145, 2006

6. B. D. Gupta, R.A. Mehta, M. M. Trangadia, "Profile of Deaths due to Electrocution: A Retrospective Study", Journal of the Indian Academy of Forensic Medicine, Vol. 34, No. 1, Jan- March 2012.

7. Z. H. Bohari, M. F. Sulaima, M. N. M. Nasir,W. M. Bukhari,M. H. Jali, M. F. Baharom, "A Novel Electromagnetic Field Detector for Extremely Low Frequency Energy", The International Journal of Engineering and Science, Vol. 3, No.6, pp. 59-67, 2014.

\section{AUTHOR PROFILE}

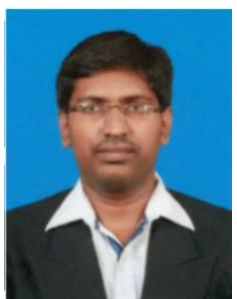

Ramesh Komarasamy was born in Tamilnadu on 1981. He received B.E. degree in Electrical and Electronics Engineering from K.S.R.College of Technology, Tamilnadu, India in 2002, and M.E. in Applied Electronics from Kongu Engg. College, Anna University, Chennai in 2005 and also received the MBA in Systems from PRIDE, Salem (Tamilnadu), India in 2005. He received his Ph.D. degree in Information and Communication Engineering from Anna University, Chennai, in 2014. At present, he is working as Professor in Kuppam Engineering College, India. He is a life member of ISTE, a member of International Association of Engineers (IAENG) and a member of International Association of Computer Science and Information Technology (IACSIT). He is acting as a reviewer in IEEE Transactions on Industrial Electronics, IETE Technical Review, Journal of Vibration and Control, Journal of Computer Science, Indian Journal of Science and Technology and he is acting as an Editor-in- Chief in Transactions on Engineering and Sciences. His fields of interest include model order reduction, controller design, optimization Techniques and renewable energy conversion systems.

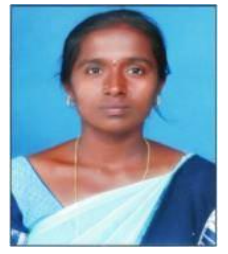

B.Archana was born in Andrapradesh on 1989. She received B.Tech.degree in Electrical and Electronics Engineering from SVPCET Andhra Pradesh, India in 2011 and M.Tech. from Kuppam Engineering College, JNT University Ananthapuram in 2018 Within the field of Electrical and Electrical Engineering now she works as Professor in Kuppam Engineering college department of electrical. She inquisitive about research and power Electronics.

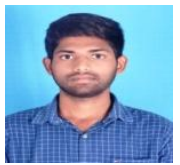

V.Tharnath Reddy was born in Andhra pradesh on 1998. He was completed intermediate education on 2016 in state board of Andhra Pradesh and also now pursuing B.Tech in Kuppam Engineering College as Electrical and Electronics Engineering.

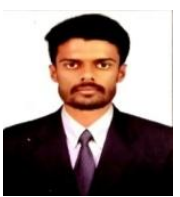

C Nagendra Kumar was born in Andhra Pradesh on 1995. He was completed intermediate education on 2012 in state board of Andhra Pradesh and also now pursuing B.Tech. in Kuppam Engineering College as Electrical and Electronics Engineering. He is a member of International Association of Engineers (IAENG).

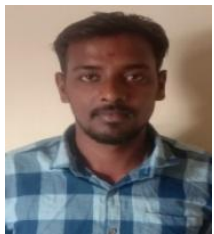

M Varaprasad was born in Andhra Pradesh on 1997. He was completed intermediate education on 2014 in state board of Andhra Pradesh and also now pursuing B.Tech.in Kuppam Engineering College as Electrical and Electronics Engineering.

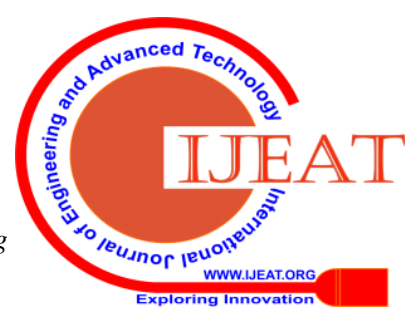

